\title{
Proton Acceleration Driven by a Nanosecond Laser from a Cryogenic Thin Solid-Hydrogen Ribbon
}

D. Margarone, ${ }^{1, *}$ A. Velyhan, ${ }^{1}$ J. Dostal, ${ }^{2}$ J. Ullschmied, ${ }^{2}$ J. P. Perin, ${ }^{3}$ D. Chatain, ${ }^{3}$ S. Garcia ${ }^{3}$ P. Bonnay,${ }^{3}$ T. Pisarczyk, ${ }_{1}^{4}$ R. Dudzak, ${ }^{2}$ M. Rosinski, ${ }^{4}$ J. Krasa, ${ }^{1}$ L. Giuffrida, ${ }^{1}$ J. Prokupek, ${ }^{2}$ V. Scuderi, ${ }^{1,6}$ J. Psikal,,${ }^{1,5}$ M. Kucharik, ${ }^{5}$ M. De Marco, ${ }^{1}$ J. Cikhardt, ${ }^{2,5}$ E. Krousky, ${ }^{2}$ Z. Kalinowska, ${ }^{4}$ T. Chodukowski, ${ }^{4}$ G. A. P. Cirrone, ${ }^{6}$ and G. Korn ${ }^{1}$

${ }^{1}$ Institute of Physics ASCR, FZU, ELI-Beamlines project, Prague 182 00, Czech Republic

${ }^{2}$ Institute of Plasma Physics ASCR, PALS Laboratory, Prague 182 00, Czech Republic

${ }^{3}$ CEA INAC-SBT, Grenoble 38000, France

${ }^{4}$ Institute of Plasma Physics and Laser Microfusion, Warsaw 01-497, Poland

${ }^{5}$ FNSPE/FEE, Czech Technical University, Prague 166 36, Czech Republic

${ }^{6}$ Laboratori Nazionali del Sud, INFN, Catania 95125, Italy

(Received 2 April 2016; revised manuscript received 27 September 2016; published 8 November 2016)

A high-power pulsed laser is focused onto a solid-hydrogen target to accelerate forward a collimated stream of protons in the range $0.1-1 \mathrm{MeV}$, carrying a very high energy of about $30 \mathrm{~J}(\sim 5 \%$ laser-ion conversion efficiency) and extremely large charge of about $\sim 0.1 \mathrm{mC}$ per laser pulse. This result is achieved for the first time through the combination of a sophisticated target system $\left(\mathrm{H}_{2}\right.$ thin ribbon) operating at cryogenic temperature $(\sim 10 \mathrm{~K})$ and a very hot $\mathrm{H}$ plasma ( $\sim 300 \mathrm{keV}$ "hot electron" temperature) generated by a subnanosecond laser with an intensity of $\sim 3 \times 10^{16} \mathrm{~W} / \mathrm{cm}^{2}$. Both the $\mathrm{H}$ plasma and the accelerated proton beam are fully characterized by in situ and ex situ diagnostics. Results obtained using the ELISE (experiments on laser interaction with solid hydrogen) $\mathrm{H}_{2}$ target delivery system at PALS (Prague) kJ-class laser facility are presented and discussed along with potential multidisciplinary applications.

DOI: 10.1103/PhysRevX.6.041030

\section{INTRODUCTION}

Hydrogen is the most abundant element in the Universe; however, it is rarely present in the solid state where it shows a hexagonal close-packed structure. Solid hydrogen was produced in the laboratory for the first time by Dewar decreasing the temperature below hydrogen's melting point ( $\sim 14 \mathrm{~K})[1]$. The interior of gas giant planets (e.g., Jupiter) is at extremely high pressure, and this condition allows for the conversion of solid molecular hydrogen $\left(\mathrm{H}_{2}\right)$ into its metallic form. Although it was predicted over 70 years ago [2], the observation of the insulator-to-metal transition in solid $\mathrm{H}_{2}$ still remains one of the great challenges of condensed-matter physics [3]. The first combination of cryogenic solid $\mathrm{H}_{2}$ and intense laser pulses is reported in Ref. [4], where isolated filaments of solid hydrogen (few $\mathrm{mm}$ in diameter and few $\mathrm{mm}$ long) were irradiated with a laser and generated a plasma with an electron energy of about $200 \mathrm{eV}$ and proton energy of about $1 \mathrm{keV}$. Other experiments were performed using a thick solid-hydrogen target irradiated by 18-ns ruby laser pulses generating $\mathrm{H}$ plasma in the backward direction [5]. Cryogenic targets have

\footnotetext{
*Corresponding author. daniele.margarone@eli-beams.eu

Published by the American Physical Society under the terms of the Creative Commons Attribution 3.0 License. Further distribution of this work must maintain attribution to the author(s) and the published article's title, journal citation, and DOI.
}

Subject Areas: Plasma Physics

also been widely used for the generation of laser-driven extreme ultraviolet (XUV) sources for lithography and microscopy [6].

In view of the prospects offered by emerging acceleration mechanisms and the motivations provided by important applications, including the potential use in hadron therapy of cancer or fast ignition for inertial confinement fusion, laserdriven proton acceleration has attracted growing attention from the scientific community $[7,8]$. Target normal sheath acceleration (TNSA), which is the most experimentally investigated laser acceleration mechanism, currently enables the production of proton beams with maximum energies of 60-85 MeV [9]. The beams typically have broad energy spectra; however, monoenergetic features in laser-driven proton beams have been shown experimentally [10].

Multidisciplinary applications, especially medical ones, have stringent requirements for laser-accelerated protons, such as high-energy (60-250 MeV), high average current (in principle achievable by using $1-10-\mathrm{Hz}$ lasers), target purity, repeatable generation, and monochromaticity. While the progress towards such requirements will be facilitated by the development of next-generation laser facilities, equally important advances can be made by developing novel target media specifically tailored to advanced acceleration regimes. The characteristics required of such a target are that it be thin $(1-50 \mu \mathrm{m})$, relatively low density $\left(5 \times 10^{20}-5 \times 10^{22} \mathrm{~cm}^{-3}\right)$ and capable of producing only protons (with no contaminants) and of operating at a high repetition rate as both refreshable and debris free. For this 
reason, cryogenic hydrogen is currently being considered and several approaches are being explored and developed ranging from in situ growth of solid-hydrogen slabs (which are typically millimeters thick and require controlled thermal evaporation to access thinner target regimes) [11] to cylindrical jets of few $\mu \mathrm{m}$ in diameter [12].

The ELISE (experiments on laser interaction with solid hydrogen) target delivery system, which is the result of a joint development carried out at CEA Grenoble, employs an alternative approach that provides a continuous flow of thin laminar targets of solid hydrogen with a very well controlled and tunable thickness in the tens of $\mu \mathrm{m}$ range [13]. This type of sophisticated target system is used here for the first time with a high-power laser (nanosecond and low contrast) to accelerate proton beams propagating forward with a maximum energy of $\sim 1 \mathrm{MeV}$ and with a total charge on the order of $0.1 \mathrm{mC}$ per laser pulse, thus demonstrating a very high laser-ion conversion efficiency ( $\sim 5 \%$ in the $0.1-1 \mathrm{MeV}$ range), which is twice that of the total charge and efficiency of plastic targets irradiated in similar experimental conditions. Besides the energy and number of accelerated protons, our measurements aim to characterize, for the first time, the interaction of a highenergy (kJ-class), long (subnanosecond), low-intensity contrast $\left(10^{-6}-10^{-1}\right.$ at $1.3-0.3$ ns prior the main pulse intensity) with a solid-hydrogen planar target. The results achieved in the experimental campaign at PALS (Prague) are extensively described and discussed. Particular attention is paid to technological issues that would be encountered in a high-energy Petawatt (PW) system [e.g., tolerance to electromagnetic pulse (EMP), vacuum compatibility, effects of prepulse] along with the future possible usage of ELISE at emerging PW-class, high repetition rate laser facilities.

\section{CRYOGENIC SOLID HYDROGEN THIN RIBBON}

The ELISE cryogenic target delivery system designed and developed at the Low Temperature Laboratory of CEA enables the production of the continuous flow of a solid- $\mathrm{H}_{2}$ slab (ribbon) through a newly designed extrusion apparatus that does not contain any movable parts. The thermodynamic properties of a $\mathrm{H}_{2}$ fluid are used to generate a pressure rise in a cell and push the solid $\mathrm{H}_{2}$ out through a calibrated nozzle. The solid- $\mathrm{H}_{2}$ ribbon has a 1-mm width, controllable thicknesses (20-100 $\mu \mathrm{m})$ depending on the nozzle employed, and an electron density of $5.36 \times 10^{22} \mathrm{~cm}^{-3}$ (steplike).

The experimental cell for the solid- $\mathrm{H}_{2}$ ribbon production is equipped with two heat exchangers, one situated at its base (E1) and another one situated at its top (E2), enabling the cell to achieve the required temperature values, as schematically shown in Fig. 1(a). The temperature of E1 $\left(T_{1}\right)$ is regulated below the triple point of the gas (i.e., below $13 \mathrm{~K}$ for $\left.\mathrm{H}_{2}\right)$ and the temperature of $\mathrm{E} 2\left(T_{2}\right)$ is regulated above it (typically $20 \mathrm{~K}$ ). When the valve V1 is opened, $\mathrm{H}_{2}$ enters the cell and immediately condenses, thus blocking the extrusion nozzle. The cell is completely filled

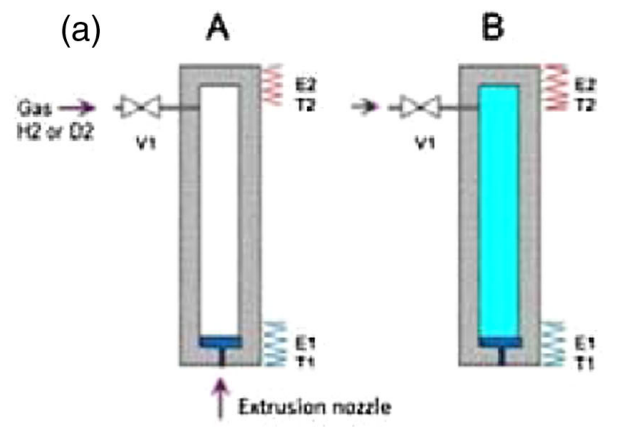

(b)

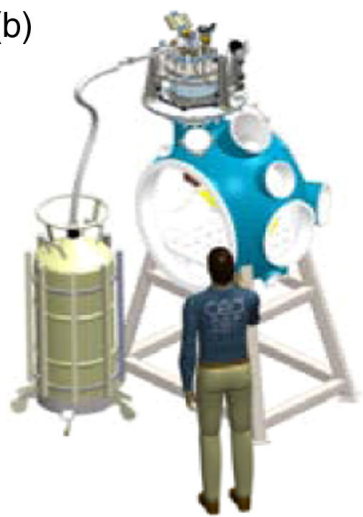

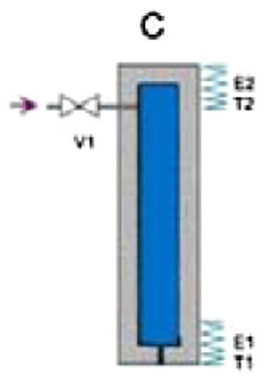
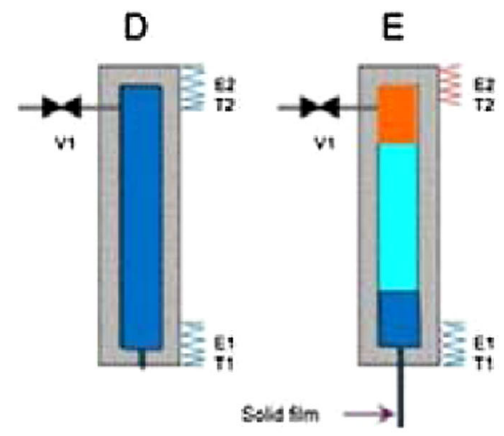

(c)

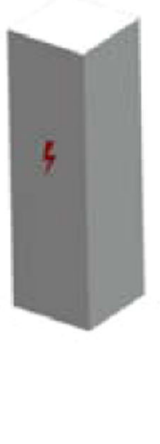

FIG. 1. (a) Different phases of the solid $\mathrm{H}_{2}$ ribbon generation, (b) scheme of the ELISE installation at PALS, (c) sketch of the ELISE nozzle. 
with liquid hydrogen $\left(T_{2}\right.$ is regulated above the triple point), then $T_{2}$ is decreased slowly below the triple point in order to fill the cell completely with solid $\mathrm{H}_{2}$ and the valve is closed. Finally, $T_{2}$ is increased to generate a pressure rise in the cell ( $T_{1}$ is regulated close to the triple point) and solid $\mathrm{H}_{2}$ is extruded through the nozzle. The solid $\mathrm{H}_{2}$ extrusion speed can be regulated close to $10 \mathrm{~mm} / \mathrm{s}$ by precisely controlling $T_{1}$ and $T_{2}$. The solid film then evaporates under the effect of heat radiation, being finally evacuated by a pumping system. More details about the ELISE working principle and functional tests performed at CEA are reported in Ref. [13].

The main parts of the ELISE installation are shown in Fig. 1(b): (i) a cryostat cooled with liquid helium through a transfer line connected to a liquid helium Dewar, (ii) an electrical cabinet situated close to the experimental chamber, (iii) an ad hoc electrical shielding system to prevent disturbances from EMP during high-energy laser-matter interaction, and (iv) a differential pumping system $\left(10^{-5}-10^{-4} \mathrm{mbar}\right)$. The aperture of the cone close to the nozzle is $38 \mathrm{deg}$ on both sides (incoming laser and outgoing proton beam) as shown in Fig. 1(c).

\section{EXPERIMENTAL SETUP AT PALS SUBNANOSECOND LASER FACILITY}

The experiment is performed at the Prague Asterix Laser System (PALS) facility in Prague [14], using the kJ-class (up to $600 \mathrm{~J}$ in our experiment), subnanosecond (0.3-ns FWHM), and linearly polarized laser beam. The laser pulse is focused into a focal spot of $80 \mu \mathrm{m}$ by an $f=2$ spherical lens to reach a nominal intensity of $3 \times 10^{16} \mathrm{~W} / \mathrm{cm}^{2}$ on the target in vacuum conditions of $10^{-5}-10^{-4}$ mbar. The solid$\mathrm{H}$ ribbon ( $62 \mu \mathrm{m}$ thick, $1 \mathrm{~mm}$ wide) is irradiated by the laser with normal incidence to the ribbon surface. The ns-long laser pulse temporal shape reported in Fig. 2(a) shows that although approximately $500 \mathrm{~J}$ are contained in the main pulse ( 300 ps at FWHM), the residual laser energy (about $100 \mathrm{~J}$ ) is stored in the nanosecond pedestal. Thus, around $50 \mathrm{~J}$ of the pulse energy is delivered on target before the maximum intensity peak is reached and, as a consequence, generates a typical preplasma. Figure 2(a) shows the presence of three intensity regions: the 0-I region represents the low-intensity $\left(3 \times 10^{10} \mathrm{~W} \mathrm{~cm}^{-2}\right)$ laser precursor, I-II is the medium intensity (up to $10^{15} \mathrm{~W} \mathrm{~cm}^{-2}$ ) pedestal, and II-III is the high-intensity (nominally up to $3 \times 10^{16} \mathrm{~W} \mathrm{~cm}^{-2}$ ) main pulse.

Besides setting up plasma and ion diagnostics, a series of basic experimental tests (vacuum and electrical) are performed to check the working functionalities of the ELISE target delivery system when operating in a harsh laser plasma environment. In fact, high-energy laser-matter interaction typically generates a giant EMP associated with electron oscillations on a target inside the PALS vacuum chamber [15], which can cause several issues for real-time detectors commonly used for laser-generated plasma
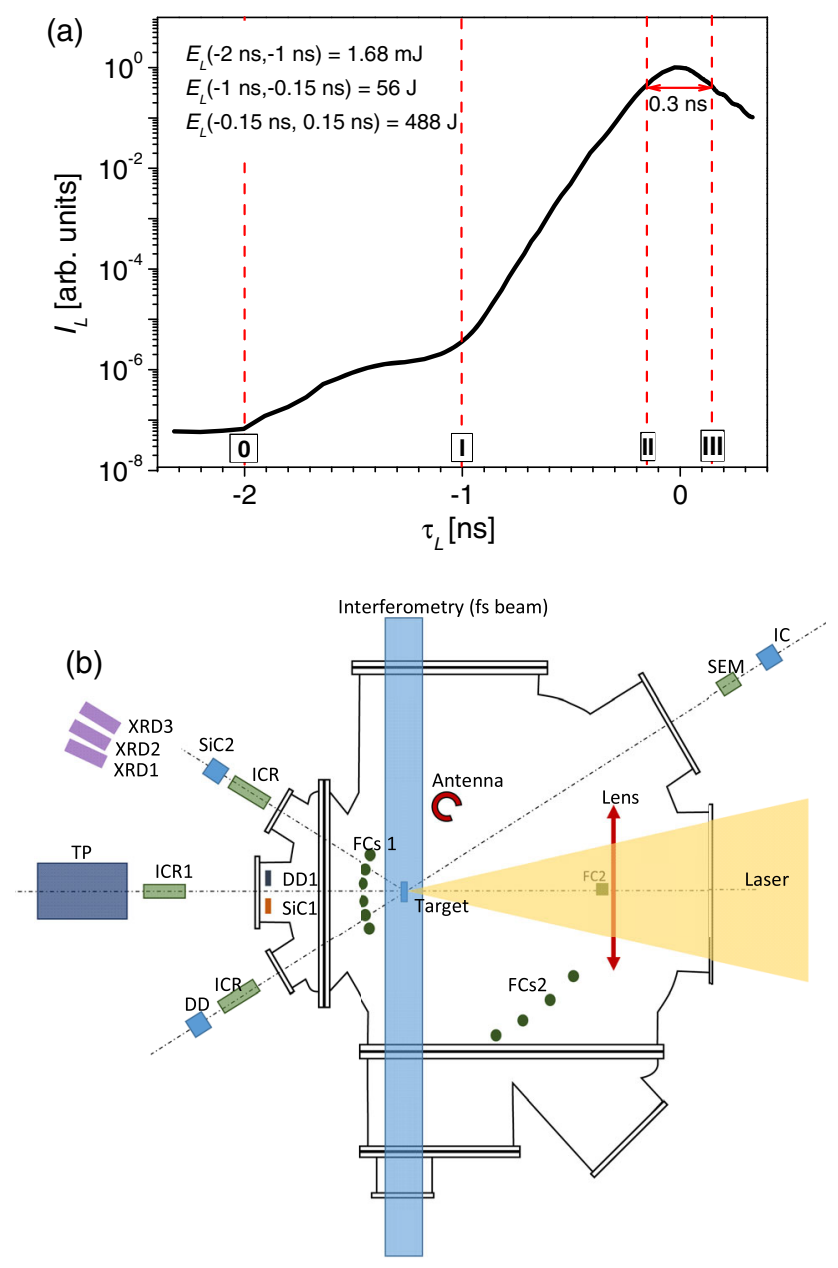

FIG. 2. (a) PALS laser temporal profile: intensity $\left(I_{L}\right)$ in arbitrary units (the laser-energy $E_{L}$ content in different time windows is reported in the caption) versus time $\left(\tau_{L}\right)$. (b) Sketch of the setup used in the experiment with the $\mathrm{H}$ target (top view).

characterization. A typical EMP signal detected by a $B$ probe (placed $26 \mathrm{~cm}$ from the target and registered with a $6-\mathrm{GHz}$ oscilloscope having 25-GS/s sampling rate) is shown in Fig. 3(a). The signal's maximum amplitude is about $130 \mathrm{~V}$ and is reached at the beginning of the lasertarget interaction. The fast Fourier transform (FFT) of the EMP signal reported in Fig. 3(b) shows a number of frequency domains ranging from $100 \mathrm{MHz}$ to $3 \mathrm{GHz}$. Although various electronic components are part of the ELISE system, the giant EMP signal amplitude did not interfere with the correct operation of the cryogenic device, mainly thanks to the optimal electrical shielding design.

Finally, the pressure value in the vacuum chamber is monitored during ELISE continuous operation and the vacuum level is found to be fully compliant with the experimental needs, especially in terms of required pressure for typically used ion diagnostics $\left(10^{-5}-10^{-4} \mathrm{mbar}\right)$.

Ion analyzers, such as a calibrated Thomson-parabola (TP) spectrometer (equipped with imaging plates), ion collectors (IC or ICR) [16], silicon-carbide (SiC) detectors, 

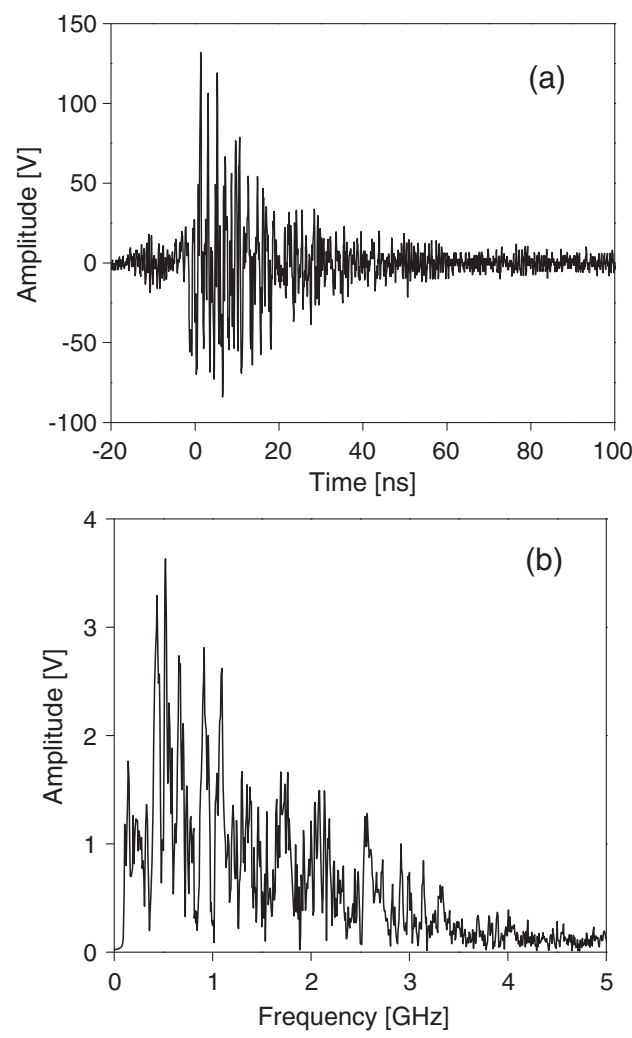

FIG. 3. Amplitude of the EMP signal measured with a $B$ probe placed $26 \mathrm{~cm}$ from the laser-target interaction point (a) and corresponding frequency spectrum (b).

and diamond detectors (DD) [17], as well as small Faraday cups (FC) [18], placed at various distances and directions, as schematically shown in Fig. 2(b), are used to detect and characterize the accelerated proton beams in terms of energy and angular distributions.

Figure 4(a) shows a typical TP spectrometer snapshot where protons, carbon ions, and other contaminants (oxygen, nitrogen, etc.) are detected when a plastic target $\left(\mathrm{CH}_{2}\right)$ is irradiated with the PALS laser. In contrast, only protons (pure $\mathrm{H}$ plasma coming from the high purity of the $\mathrm{H}_{2}$ gas) are accelerated from the $\mathrm{H}$ target, as reported in Fig. 4(b). This TP spectrum confirms that the cryogenic $\mathrm{H}$ target and, as a consequence, the produced hydrogen plasma are free of any contaminants that are present on the surface of metallic or plastic foils typically used in the TNSA acceleration regime. The TOF spectra measured for the same laser shot with the $\mathrm{H}$ target by $\mathrm{SiC}$ and IC are shown in Fig. 4(c), along with the TP signal recalculated in $\mathrm{TOF}$ at an equivalent detection distance of $1.75 \mathrm{~m}$. The $\mathrm{SiC}$ and the DD are more suitable to detect fast (energetic) protons as described in Ref. [17] compared to IC; in fact, the TOF signal around $200 \mathrm{~ns}$ [see Fig. 4(c)] is much more pronounced in the case of SiC. The photopeak ascribable to the XUV plasma radiation (present in all the used detectors) is measured almost instantaneously and is used as a trigger signal.
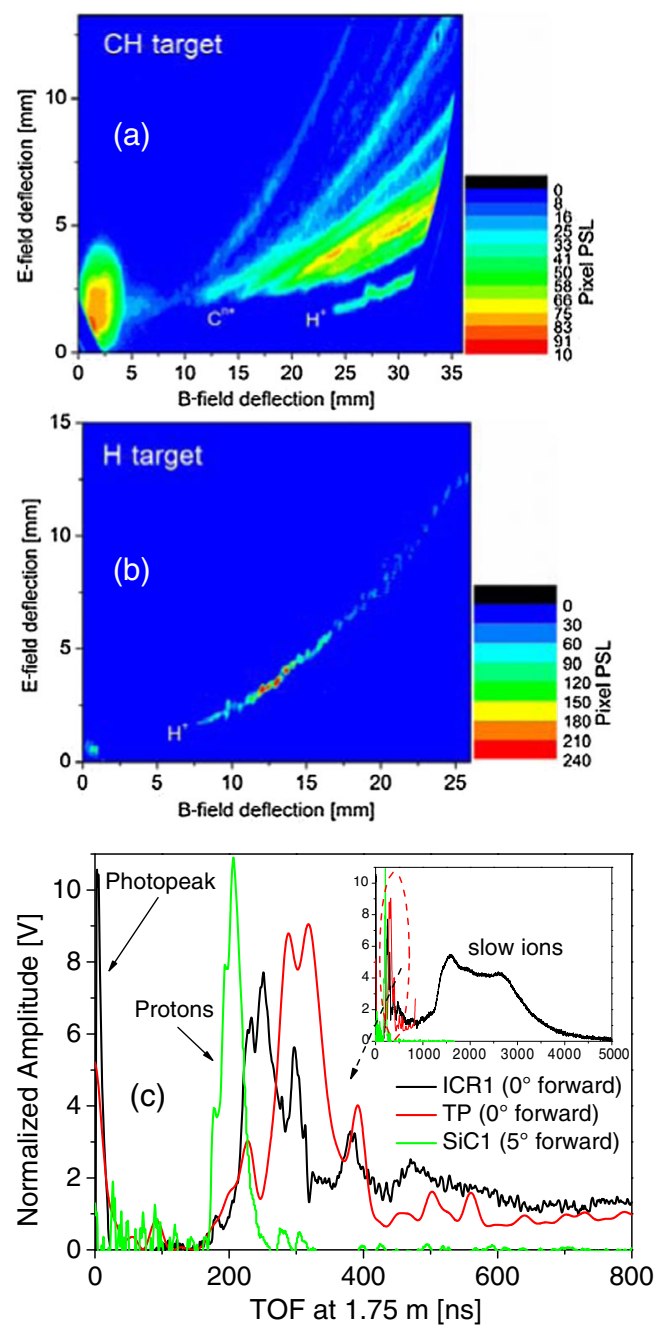

FIG. 4. (a) TP snapshot showing the typical signal from a plastic target (presence of protons, carbon, and other ions), where PSL is the photo-stimulated luminescence value. (b) TP snapshot showing the exclusive presence of protons for the $\mathrm{H}$ target (pure H plasma). (c) TOF spectra detected by ICR1, SiC1, and TP (recalculated at a distance of $1.75 \mathrm{~m}$ ). The inset shows the slow ion TOF signal present up to $5 \mu \mathrm{s}$.

A Ti:sapphire laser system having a wavelength of $808 \mathrm{~nm}$ and pulse duration of $40 \mathrm{fs}$ is used to carry out time-resolved measurements that enable the determination of plasma density distribution through a three-frame interferometric system previously optimized and normally used at PALS [19]. The three frames are recorded successively with a time delay of $1 \mathrm{~ns}$. The 2D plasma density profiles are used to measure the plasma electron density and, as a consequence, the number of slow protons in the laser produced plasma propagating both forward and backward.

Three scintillation detectors for hard $\mathrm{x}$ rays (HXR1, HXR2, HXR3) are placed $3 \mathrm{~m}$ from the target and at $45 \mathrm{deg}$ with respect to the target normal in the forward direction, as sketched in Fig. 2(b). These detectors are described in detail in Ref. [20]. The HXR1-3 detectors are shielded by 
interlocked lead bricks of $5 \mathrm{~cm}$ thickness, and by 5,10 , and $15 \mathrm{~cm}$ of lead shield at their front, respectively. Moreover, since the sensitivity of each detector is slightly different, they are cross-calibrated using the same $\mathrm{Pb}$ shielding $(5 \mathrm{~cm})$ in front of all detectors in a few dedicated shots. The HXR measurements are important for the estimation of the socalled "hot electron" temperature assuming that hard $\mathrm{x}$ rays are produced as bremsstrahlung radiation of such electrons, as described in Ref. [21].

\section{RESULTS AND DISCUSSION}

Four raw interferometric snapshots recorded through the optical interferometry technique, using the fs-class Ti: sapphire laser as a probe beam, are reported in Fig. 5. They show the H-plasma expansion for the same laser shot at four different times with respect to the arrival of the main laser pulse: $-0.2 \mathrm{~ns}$ [Fig. 5(a)] and -0.07 ns [Fig. 5(b)] before the main intensity peak, 0.9 ns [Fig. 5(c)] and 1.9 ns [Fig. 5(d)] after it. The interferometry snapshots [Figs. 5(b)-5(d)] are acquired during the same laser shot, while Fig. 5(a) is acquired during a different laser shot but at very similar laser irradiation conditions. The plasma electron density maps can be retrieved after a proper analysis using the "maximum-of-a-fringe" method [19,22], as shown in Figs. 5(i) $-5(\mathrm{n})$ for the four different time frames; thus, the corresponding axial density profile can be calculated [see plots in Figs. 5(i) and 5(1)-5(n)]. The plasma density $\left(n_{e}\right)$ profile on the $z$ axis roughly follows an exponential decay function $\left(n_{e}(z)^{1 / 4} n_{0} e^{-z / L}\right)$, where $L$ is the density scale length and $n_{0}$ is the maximum electron density $[19,22]$. The interferometric measurements show the presence of a preplasma generated on the target front side at about -200 ps (i.e., before the main intensity peak) with a scale length of about $150 \mu \mathrm{m}$. At about $-70 \mathrm{ps}$, a plasma is also generated at the target rear side with a scale length of $200 \mu \mathrm{m}$. The plasma length at the target rear side after 0.9 and $1.9 \mathrm{~ns}$ is approximately 600 and $900 \mu \mathrm{m}$, respectively, which corresponds to a bulk plasma expansion velocity of $\sim 5 \times 10^{5} \mathrm{~m} / \mathrm{s}$, which identifies the group of slow protons in the $\mathrm{H}$ plasma. The group of fast protons with kinetic energy in the range $0.1-1 \mathrm{MeV}$ is not discernable. The estimated plasma electron number, which basically corresponds to the total number of slow protons, is $4 \times 10^{16}$ at the front and $3 \times 10^{16}$ at the rear side of the irradiated target after $1.9 \mathrm{~ns}$ with respect to the arrival of the main laser pulse.

The normalized TOF proton signals measured through different ion detectors are summarized in Fig. 6(a). Both ICR1 and TP (placed along the laser axis in the forward direction) show a proton current signal starting slightly after $100 \mathrm{~ns}$, which corresponds to proton energy of approximately 1.2 MeV. On the other hand, DD1 (placed at $5 \mathrm{deg}$ from the laser axis in the forward direction) shows a slightly faster proton signal corresponding to proton
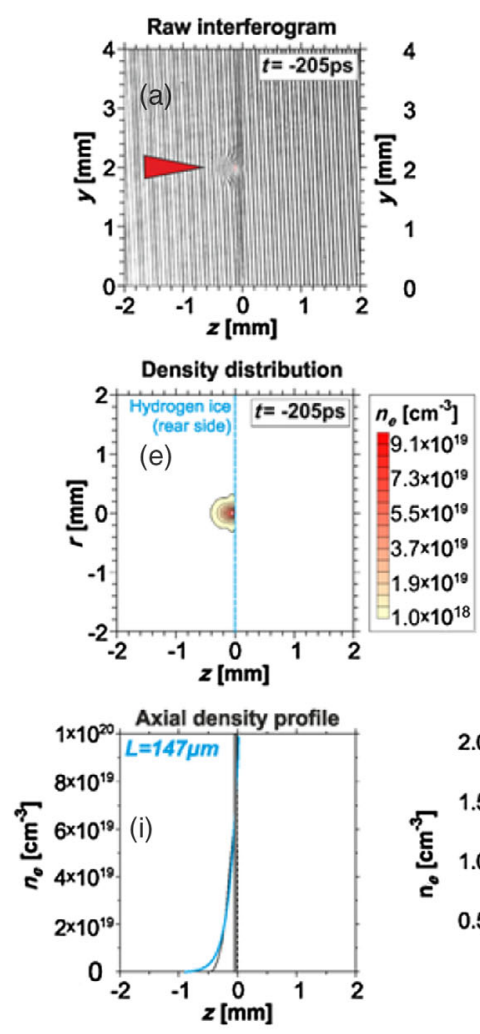
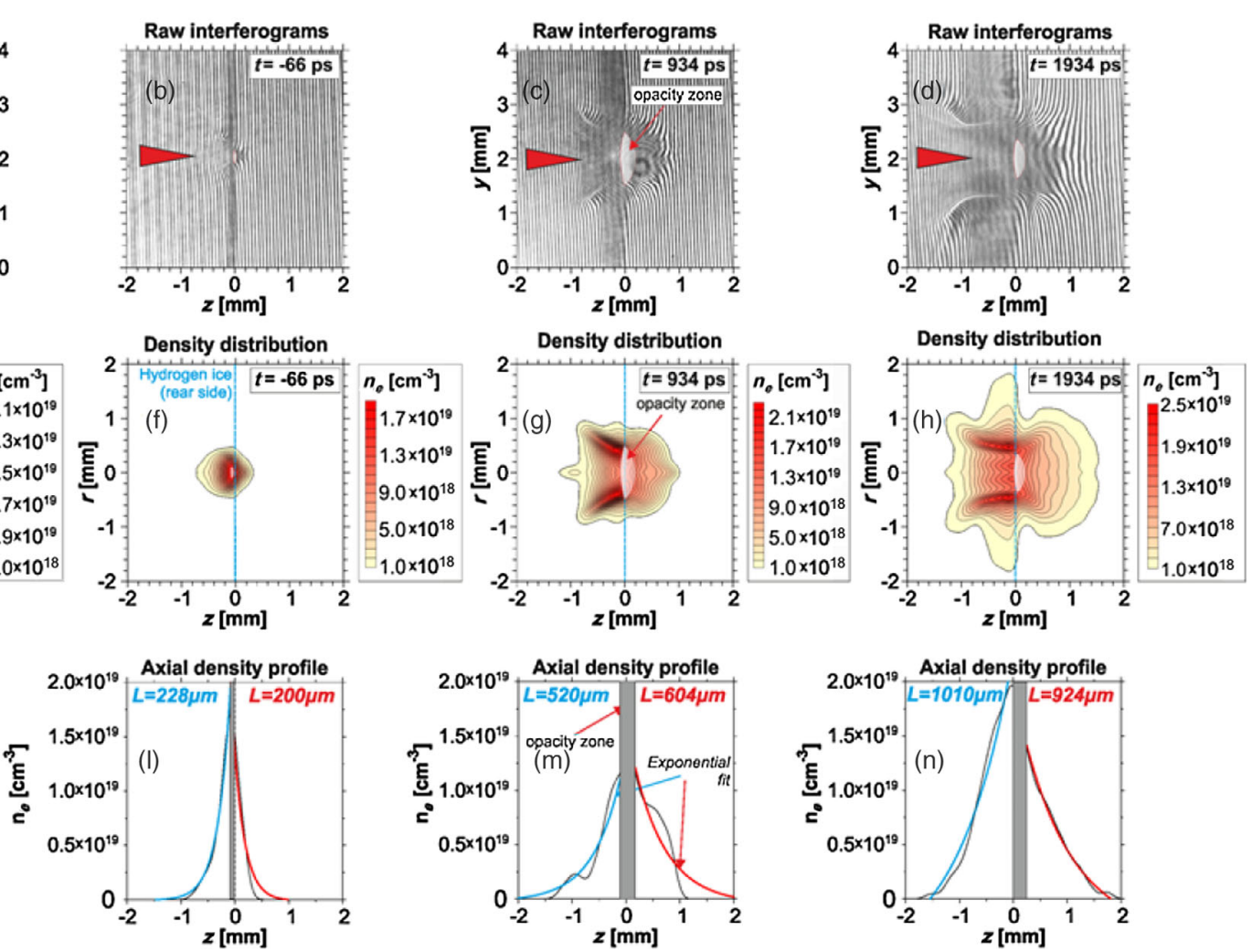

FIG. 5. Raw interferometry snapshots recorded at $-0.2,-0.07,0.9$, and $1.9 \mathrm{~ns}$ (a)-(d), respectively, corresponding plasma density maps (e)-(h), and axial density profiles (i)-(n). The laser propagates from left to right, as shown by the red arrow. 

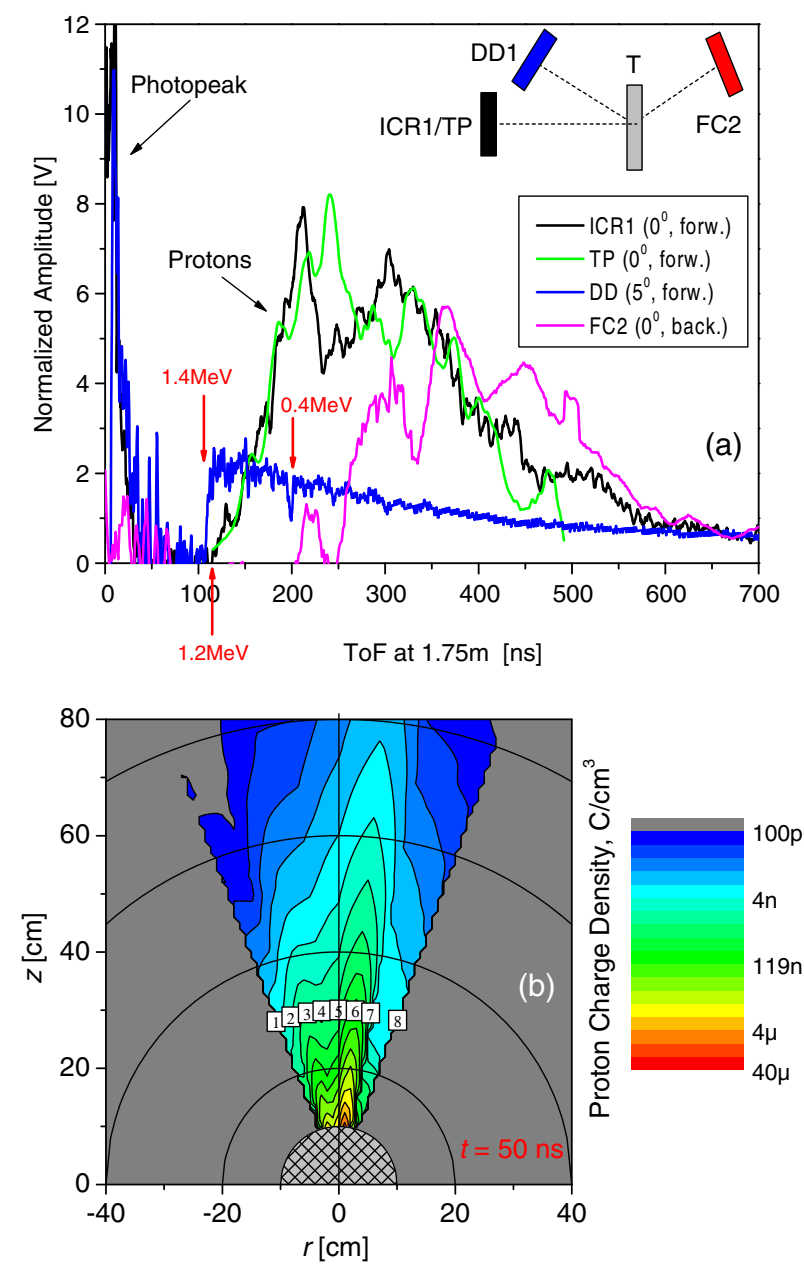

FIG. 6. (a) Normalized TOF signal for the different ion detectors (ICR1, TP, DD1, FC2) used during the same laser shot. (b) Proton angular distribution reconstructed from the eight FCs placed in the forward direction (shown as white squares).

energy of about $1.4 \mathrm{MeV}$. FC2 (placed at 5 deg from the laser axis in the backward direction) shows a much slower TOF signal at around $200 \mathrm{~ns}$, which indicates proton energy of about $0.4 \mathrm{MeV}$, thus with a maximum kinetic energy clearly lower than the proton stream propagating forward.

Figure 6(b) shows the proton angular distribution in terms of charge density $\left(\mathrm{C} / \mathrm{cm}^{3}\right)$, which is estimated through the eight FCs placed at various angles in the forward direction [white squares in Fig. 6(b)]. The charge density is derived from the measured ion currents, as presented in Ref. [23]. The maximum ion aperture cone is approximately $25 \mathrm{deg}$, as shown in the inset of Fig. 7(a), and a small deflection of $\sim 5 \mathrm{deg}$ in the ion stream propagation (with respect to the laser axis) is measured. In fact, the difference shown in the beginning of the proton signal by DD1 compared to ICR1 or TP seems to be ascribable to the abovementioned deflection in the ion emission, since the high-proton energies have a privileged direction of propagation (in this case $\sim 5 \mathrm{deg}$ from the laser axis).
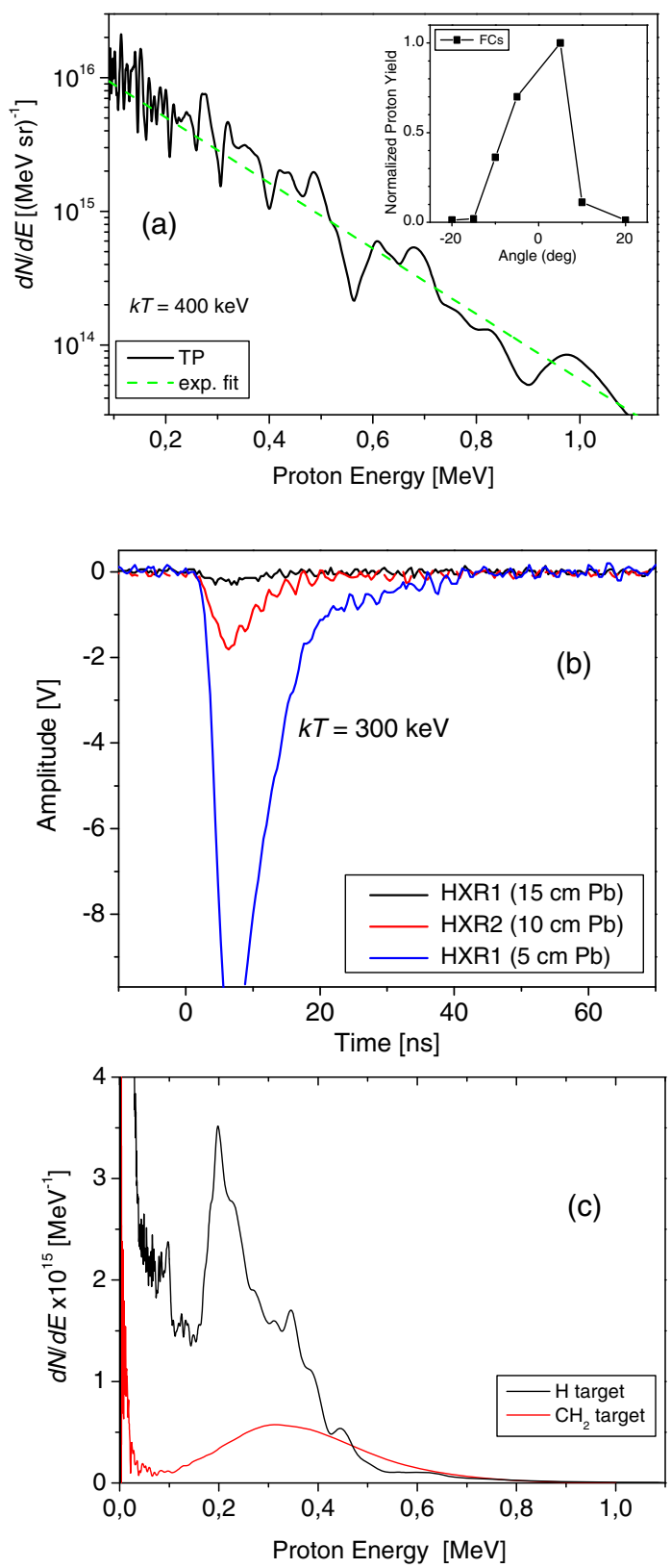

FIG. 7. (a) Proton energy distribution measured with TP (black line) and linear fit (dashed green line) along with the ion angular distribution measured with FCs (see inset). (b) HXR signals measured with the three detectors having different $\mathrm{Pb}$ filter thickness (the signal recorded by the detector with a $5-\mathrm{cm} \mathrm{Pb}$ shielding is saturated; however, an extrapolation of the signal is used to calculate the hot electron temperature). (c) Proton energy distribution recalculated from TOF spectra acquired with solid-H and $\mathrm{CH}_{2}$ targets in similar laser irradiation conditions.

The TP spectrum is converted in proton energy distribution as reported in Fig. 7(a). A cone of $\sim 25$ deg for the accelerated proton stream angular distribution is measured by the small FCs placed behind the target [shown in the inset of Fig. 7(a)]. The $\gamma$-ray signals measured by the HXR plastic scintillators (shielded with three different $\mathrm{Pb}$ filters) 

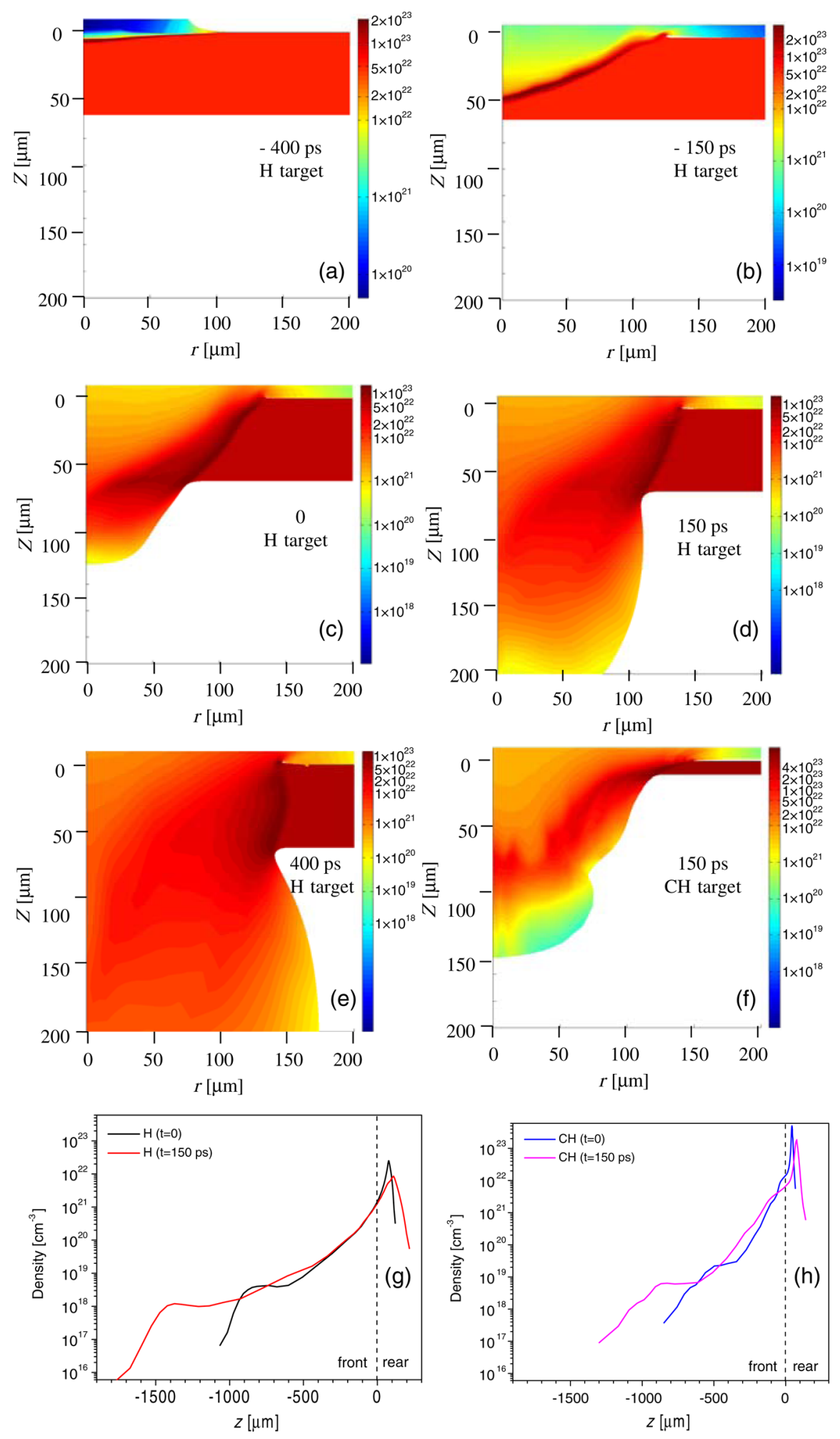

FIG. 8. Hydrodynamic simulation outputs showing the electron density profiles for the $\mathrm{H}$ target at different times after the main laser peak intensity (a)-(e) and for the $\mathrm{CH}$ target at $150 \mathrm{ps}$ (f). The laser beam propagates from the top along the $z$ axis. The electron density profiles along the $z$ axis at 0 and $150 \mathrm{ps}$ are shown in (g) and (h), respectively, for the $\mathrm{H}$ and $\mathrm{CH}_{2}$ targets. 
for the same laser shot are shown in Fig. 7(b). The signal recorded by the detector with a $5-\mathrm{cm} \mathrm{Pb}$ shielding is saturated; however, an extrapolation of the signal is used to calculate the hot electron temperature $\left(k T_{\text {hot }}\right)$. The temperature estimated from the HXR spectra analysis is approximately $300 \mathrm{keV}$, which is in good agreement with the value calculated through the exponential fit of the TP proton energy distribution $(\sim 400 \mathrm{keV})$ shown as a dashed line in Fig. 7(a). This hot electron temperature estimation fits very well the measured maximum proton energy $\left(E_{\max } \sim 1.4 \mathrm{MeV}\right)$, as predicted by experimental scaling laws $\left(E_{\max } \sim 4.5 k T_{\text {hot }}\right)$ reported in Ref. [24].

The maximum proton energy measured with the solid- $\mathrm{H}$ target is in agreement with our previous results using metallic, semiconductor, and plastic foils at PALS [25]. However, the number of protons propagating forward with energy of $0.1-1 \mathrm{MeV}$ is enhanced by about a factor of 3 (from $2 \times 10^{14}$ to $6.5 \times 10^{14}$ ) compared to a plastic $\left(\mathrm{CH}_{2}\right)$ target having a thickness of $10 \mu \mathrm{m}$, which roughly corresponds to the areal density of the used $\mathrm{H}$ target. The proton number is enhanced by about a factor of 4 for protons having energies between 150 and $400 \mathrm{keV}$ (from $1.1 \times 10^{14}$ to $4.8 \times 10^{14}$ ), as clearly shown in Fig. 7(c). The estimated total energy for the collimated proton beam with energies of $0.1-1 \mathrm{MeV}$, which are accelerated by the subnanosecond laser into the forward direction, is about $30 \mathrm{~J}$, and the corresponding laser-proton acceleration efficiency $\eta$ (ratio between the total proton beam energy and the incoming laser energy) is about $5 \%$.

We carry out hydrodynamic simulations for both the $\mathrm{H}$ and $\mathrm{CH}_{2}$ targets, in order to obtain important information about the interaction physics and the peculiarity in laser ablation with the given temporal profile provided by the PALS system having a low laser intensity contrast [see Fig. 2(a)]. Such simulations are mainly used to model the evolution of the plasma electron density, especially at early stages of the laser-matter interaction. However, hydrodynamic simulations cannot properly describe the acceleration mechanism close to the main laser peak intensity $\left(>10^{16} \mathrm{~W} / \mathrm{cm}^{2}\right)$ where hot electrons (responsible for the acceleration of protons with the highest energies) are generated and kinetic effects (potentially responsible for laser absorption enhancement) are expected. The 2D PALE (Prague arbitrary LagrangianEulerian) hydrodynamic code working in cylindrical geometry is used [26]. This code employs the compatible staggered Lagrangian numerical scheme [27], the quotidian equation of state (QEOS), and a rezoning or remapping mechanism improving the robustness of the simulations [28]. The thermal conductivity is treated using a mimetic method with the standard Spitzer-Harm heat conductivity coefficient and heat flux limiter. Ray tracing is used for the laser absorption modeling. A thickness of $10 \mu \mathrm{m}$ is chosen for the $\mathrm{CH}_{2}$ target to keep the same areal density as the $62-\mu \mathrm{m}$-thick $\mathrm{H}$ target. The outputs of our hydrodynamic simulations are shown in Fig. 8. The maximum electron density for the $\mathrm{H}$ plasma is more than a factor of 20 lower than the one of the $\mathrm{CH}$ plasma during the whole laser interaction. Furthermore, the electron density values at the focal plane $(\mathrm{FP}=0)$ at two different times for both targets show an enhancement of the relative ratio between the $\mathrm{CH}$ - and the $\mathrm{H}$-plasma densities from 10 at the main peak intensity $(t=0)$ to 5 at the FWHM value of the laser intensity ( $t=150 \mathrm{ps}$ ). In particular, the $\mathrm{CH}$-plasma electron density at the focal plane (where the laser intensity is maximized) is about $1.3 \times 10^{22}$ and $6 \times 10^{21} \mathrm{~cm}^{-3}$ at 0 and $150 \mathrm{ps}$, respectively. On the other hand, the H-plasma electron density at the focal plane is about $1.3 \times 10^{21} \mathrm{~cm}^{-3}$, independent of time, thus showing that the laser pulse constantly interacts with a "quasisteady" near-critical density plasma (approximately 2 times the critical density), while the $\mathrm{CH}$ plasma always shows an overcritical density, even for longer times. Such numerical results suggest a potential enhancement of the laser absorption at the highest intensities $\left(>10^{16} \mathrm{~W} / \mathrm{cm}^{2}\right)$, which involves a larger plasma volume in the case of the $\mathrm{H}$ target, thus leading to a higher proton acceleration efficiency, as measured experimentally. On the other hand, in the case of the $\mathrm{CH}_{2}$ target, the laser is entirely absorbed at the target front side.

\section{CONCLUSIONS AND PERSPECTIVES}

In this paper, we present and discuss the first experimental results obtained through irradiating a cryogenic thin $(62-\mu \mathrm{m})$ solid-hydrogen ribbon with a high-power laser. The investigation of such a system is potentially interesting for condensed-matter physics, e.g., through $\mathrm{H}$ spectroscopy studies at extreme conditions (high pressure and high temperature gradient) [29].

The generation of pure hydrogen plasma has advantages from the experimental point of view in terms of plasma generation and characterization, as well as for future multidisciplinary applications of laser-accelerated proton beams, since such a target delivery system can potentially be used at high repetition rates and is essentially debris free. Furthermore, clear benefits for theoretical modeling and numerical simulations are evident. In fact, $\mathrm{H}$ plasma can be considered as an ideal sample for theoretical investigations and their experimental verification both in terms of accuracy (a lower number of approximations is needed) and also reduced computational time for modeling [especially with particle-in-cell (PIC) codes for femtosecond and picosecond laser-matter interaction].

This target delivery system has been used on the PALS laser (long pulse, high energy but moderate intensity), which is not the ideal system for reaching high proton energies; however, using ELISE on this laser system requires the solution of similar technological issues that would be encountered on a high-energy PW system (e.g., tolerance to EMP, vacuum compatibility, effects of prepulses). Thin ribbons down to $25 \mu \mathrm{m}$ have already been produced; however, the stability of the target along the laser 
propagation axis has to be improved in terms of shot-to-shot fluctuations, for instance, using a simple technical solution where a mechanical "guide" is placed under the nozzle in order to "channel" the $\mathrm{H}$ ribbon and to limit its oscillations. Nevertheless, the shot-to-shot fluctuations measured in our experiment ( $\pm 25 \mu \mathrm{m}$ along the laser axis) do not affect our experimental results; in fact, we estimate a very small difference in the angle between the laser axis and the target normal due to such spatial fluctuations $\left(<0.4^{\circ}\right)$, which does not affect the proton beam features in terms of directionality and maximum energy. The technological solutions employed to operate the ELISE cryostat allow, in principle, the production of $\mathrm{H}$ ribbons with thickness down to $5 \mu \mathrm{m}$. The production of lower thickness ribbons ( $1 \mu \mathrm{m}$ or less) can potentially be achieved using a slightly different geometry where the hydrogen ribbon is partially evaporated just before the laser shot through the interaction with a synchronized low-energy laser operating at a wavelength of around $2 \mu \mathrm{m}$, which is the range of maximum absorption for hydrogen.

Our experimental achievements with the ns-class, lowcontrast PALS laser demonstrate that a very high total energy of about $30 \mathrm{~J}$ is carried by the forward accelerated collimated proton stream to which corresponds a laserproton acceleration efficiency of $\sim 5 \%$ in the $0.1-1 \mathrm{MeV}$ energy range (2-3 times higher than standard plastic foils in the same irradiation conditions), and a proton beam charge of $\sim 0.1 \mathrm{mC}$ per laser pulse. Such efficient proton acceleration is ascribable to volumetric effects in the near-critical density $\mathrm{H}$ plasma along the entire target thickness. Although so-called "collisionless shock acceleration" has been shown as a result of the interaction of 10-100-ps laser pulses with a near-critical density target [30], this regime is excluded in our case, since our experiment shows a much higher proton yield compared to Ref. [30]. Moreover, the maximum laser intensity in our experiment is relatively low (corresponding to $a_{0}=0.2$ ); thus, the resulting hole-boring velocity associated with the ponderomotive force is also low, both for the fully ionized solid density $\mathrm{H}$ target (approximately $5 \times 10^{-4} \mathrm{c}$ ) and for the H-plasma density at the laser peak intensity $\left(3 \times 10^{-3} c\right.$, where $c$ is the speed of light). This would result in proton energies below $1 \mathrm{keV}$ and approximately $20 \mathrm{keV}$, respectively, estimated by taking into account that the shock velocity is close to the hole-boring velocity [7].

Although a proton acceleration conversion efficiency of $15 \%$ was experimentally achieved by Brenner et al. for energies of 5-30 MeV using two temporally separated 800 -fs laser beams and 5- $\mu \mathrm{m}$-thick Au foil (45 deg incidence angle and $p$ polarization) [31], our experimental achievement (5\% conversion efficiency for the forward accelerated proton beam in the $0.1-1 \mathrm{MeV}$ energy range) has never been reached with ns-class lasers operating at moderate laser intensities. This result can potentially be improved upon if a ps-class laser system is used. In fact, based on our PIC simulations in the fs regime (see Fig. 9), the ion acceleration efficiency with

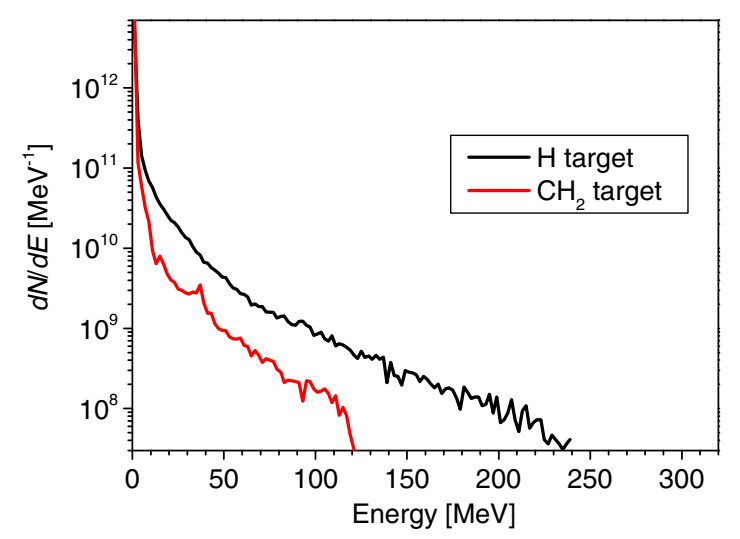

FIG. 9. Proton energy distributions calculated through PIC numerical simulations for different targets with a thickness of $5 \mu \mathrm{m}$ and for a laser intensity of $\sim 6 \times 10^{21} \mathrm{~W} / \mathrm{cm}^{2}$.

a low-density solid-H target can reach values of about $25 \%$. Thus, an approximation of $20 \%$ for the laser-to-proton acceleration efficiency can be considered if the target thickness is optimized for the specific ps-laser system temporal characteristics. In principle, our experimental achievements might support novel scenarios for the fast ignition scheme where, as suggested in Ref. [32], a collimated laser-generated proton beam is used to induce ignition in a precompressed DT fuel $\left(400 \mathrm{~g} / \mathrm{cm}^{3}\right)$ with a standard inertial confinement fusion (ICF) geometry. Various theoretical works indicate a preference for relatively low proton energies $(\sim 10 \mathrm{MeV})$ at a fuel temperature of a few $\mathrm{keV}$, having a proton bunch duration of several ps (required for the fast ignition scheme) and $\sim 10 \mathrm{~kJ}$ total proton beam energy [33]. In the case of the investigated $\mathrm{H}$ target, assuming a laserproton acceleration efficiency of $\sim 20 \%$, the required ps-class laser system should deliver energy of $\sim 50 \mathrm{~kJ}$ and moderate laser intensity on target to achieve ignition.

In order to explore the direction in which the use of a cryogenic hydrogen target could develop, especially in terms of multidisciplinary applications of laser-driven proton beams, we perform preliminary 2D PIC simulations using the EPOCH code at ultrahigh laser intensities [34]. Laser parameters relevant to the future $10-\mathrm{Hz}, \mathrm{PW}$-class, 800-nm laser system available at ELI-Beamlines [35] are used; thus, the laser peak intensity is $6 \times 10^{21} \mathrm{~W} / \mathrm{cm}^{2}$ (18-J laser energy on target, 3- $\mu \mathrm{m}$ FWHM focal spot diameter, 30-fs laser pulse duration, normal incidence irradiation angle). Fully ionized solid density $\mathrm{H}$ and $\mathrm{CH}_{2}$ targets are considered with a thickness of $5 \mu \mathrm{m}$. The cell size is $20 \times 20 \mathrm{~nm}^{2}$, and each cell contains 62 macroelectrons for the $\mathrm{H}$ foil and 400 macroelectrons for the $\mathrm{CH}_{2}$ foil. A corresponding number of macroions is used in order to ensure plasma charge neutrality; moreover, all particles have the same numerical weights. The comparison between $5-\mu \mathrm{m}$-thick solid-H foil and 5- $\mu$ m-thick plastic $\left(\mathrm{CH}_{2}\right)$ foil is shown in Fig. 9. The use of 5- $\mu \mathrm{m}$-thick solid $\mathrm{H}$ seems to cover the entire "clinical window" for cancer therapy in 
terms of proton energy (60-250 MeV), thus clearly showing the potential of this low-density cryogenic target compared to standard solid foils. Furthermore, such a target seems to be ideal for future application in laserdriven proton therapy due to the high purity and the high proton beam charge per laser pulse $(\sim 15 \mathrm{nC}$ for proton energies higher than $60 \mathrm{MeV}$ ). The clear advantage of the proposed target, which is demonstrated experimentally in the ns regime, i.e., the energy coupling into energetic particles, is also confirmed by our numerical simulations performed in the fs regime. In fact, a very high laser-toproton conversion efficiency of $\sim 25 \%$ is estimated for the $\mathrm{H}$ target for energies higher than $5 \mathrm{MeV}$, in comparison to $\sim 10 \%$ calculated for the $\mathrm{CH}_{2}$ target. This result is linked to the laser absorption along the overall target thickness, which is strongly enhanced ( $\sim 70 \%$ for the $\mathrm{H}$ target and $\sim 30 \%$ for the $\mathrm{CH}_{2}$ target). Moreover, electrons are heated more efficiently ( $\sim 25 \mathrm{MeV}$ for the $\mathrm{H}$ target and $\sim 20 \mathrm{MeV}$ for the $\mathrm{CH}_{2}$ target) within the whole $\mathrm{H}$-foil volume thanks to a more efficient hole-boring mechanism. In fact, for the $\mathrm{H}$ target, the hole-boring velocity is substantially higher due to its lower density in comparison to the $\mathrm{CH}_{2}$ foil (more than a factor of 6) and to the lower ion mass in the generated plasma (only $\mathrm{H}$ is present in the cryogenic target, but $\mathrm{C}$ is also present in the plastic foil), thus leading to a different regime of laser-target interaction and to substantial enhancement of the laser absorption.

\section{ACKNOWLEDGMENTS}

The authors acknowledge the following scientific and technical personnel who provided additional support to the different teams involved in this experimental work: M. Pfeifer, J. Skala, J.M. Mathonnet, T. Lastovicka, M. Kozlova, J. Hrebicek, J. Kovar, M. Jantac, K. Brabcova, M. De Napoli, N. Randazzo, and R. Jack. This research was mainly sponsored by the project ELI - Extreme Light Infrastructure-Phase 2 (CZ.02.1.01/0.0/0.0/15_008/0000162) through the European Regional Development Fund, by the Ministry of Education, Youth and Sports of the Czech Republic (National Program of Sustainability II Project No. LQ1606, PALS RI Projects No. LM2010014 and No. LM2015083, COST Project No. LD14089), and by the Czech Science Foundation (Projects No. 15-02964S and No. P205-11-P712). Computational resources were provided by the CESNET LM2015042 and the CERIT Scientific Cloud LM2015085, provided under the program "Projects of Large Research, Development, and Innovations Infrastructures".

[1] J. Dewar, Sur la Solidification de l'Hydrogene, Ann. Chim. Phys. 18, 145 (1899).

[2] E. Wigner and H. B. Huntington, On the Possibility of a Metallic Modification of Hydrogen, J. Chem. Phys. 3, 764 (1935).
[3] S. Deemyad and I. F. Silvera, Melting Line of Hydrogen at High Pressures, Phys. Rev. Lett. 100, 155701 (2008).

[4] P. A.H. Saunders, P. Avivi, and W. Millar, Laser Produced Plasmas from Solid Hydrogen Targets, Phys. Lett. 24A, 290 (1967).

[5] P. Mulser, R. Siger, and S. Witkowski, Plasma Production by Laser, Phys. Rep. 6, 187 (1973).

[6] F. Jin, K. Gabel, M. C. Richardson, M. Kado, A. F. Vasilev, and D. Salzmann, in Applications of Laser Plasma Radiation SPIE Proceedings Vol. 151 (SPIE-International Society for Optical Engineering, Bellingham, WA, 2015); M. Berglund, L. Rymell, H. M. Hertz, and T. Wilhein, Cryogenic Liquid-Jet Target for Debris-Free Laser-Plasma Soft X-Ray Generation, Rev. Sci. Instrum. 69, 2361 (1998); R. Lebert, G. Schriever, T. Wilhein, and B. Niemann, Soft X-Ray Emission of LaserProduced Plasmas Using a Low-Debris Cryogenic Nitrogen Target, J. Appl. Phys. 84, 3419 (1998).

[7] A. Macchi, M. Borghesi, and M. Passoni, Ion Acceleration by Superintense Laser-Plasma Interaction, Rev. Mod. Phys. 85, 751 (2013).

[8] S. V. Bulanov and V. S. Khoroshkov, Feasibility of Using Laser Ion Accelerators in Proton Therapy, Plasma Phys. Rep. 28, 453 (2002); S. V. Bulanov, J. J. Wilkens, T.Zh. Esirkepov, G. Korn, G. Kraft, S. D. Kraft, M. Molls, and V.S. Khoroshkov, Laser Ion Acceleration for Hadron Therapy, Phys. Usp. 57, 1149 (2014).

[9] R. A. Snavely, M. H. Key, S. P. Hatchett, T. E. Cowan, M. Roth, T. W. Phillips, M. A. Stoyer, E. A. Henry, T. C. Sangster, M. S. Singh, S. C. Wilks, A. MacKinnon, A. Offenberger, D. M. Pennington, K. Yasuike, A. B. Langdon, B. F. Lasinski, J. Johnson, M. D. Perry, and E. M. Campbell, Intense High-Energy Proton Beams from Petawatt-Laser Irradiation of Solids, Phys. Rev. Lett. 85, 2945 (2000); S. A. Gaillard, T. Kluge, K. A. Flippo, M. Bussmann, B. Gall, T. Lockard, M. Geissel, D. T. Offermann, M. Schollmeier, Y. Sentoku, and T. E. Cowan, Increased Laser-Accelerated Proton Energies via Direct Laser-Light-Pressure Acceleration of Electrons in Microcone Targets, Phys. Plasmas 18, 056710 (2011); F. Wagner et al., Maximum Proton Energy above $85 \mathrm{MeV}$ from the Relativistic Interaction of Laser Pulses with Micrometer Thick CH2 Targets, Phys. Rev. Lett. 116, 205002 (2016).

[10] B. M. Hegelich, B. J. Albright, J. Cobble, K. Flippo, S. Letzring, M. Paffett, H. Ruhl, J. Schreiber, R. K. Schulze, and J.C. Fernandez, Laser Acceleration of Quasimonoenergetic MeV Ion Beams, Nature (London) 439, 441 (2006); A. Henig, S. Steinke, M. Schnurer, T. Sokollik, R. Horlein, D. Kiefer, D. Jung, J. Schreiber, B. M. Hegelich, X. Q. Yan, J. Meyer-ter-Vehn, T. Tajima, P. V. Nickles, W. Sandner, and D. Habs, Radiation-Pressure Acceleration of Ion Beams Driven by Circularly Polarized Laser Pulses, Phys. Rev. Lett. 103, 245003 (2009).

[11] S. Bedacht, G. Schaumann, A. Blazevic, M. Roth, D. H. H. Hoffmann et al., GSI Scientific Report No. PNI-PP-25, 2012, p. 458.

[12] A. Propp, SLAC Technical Note No. SLAC-TN-15-078, 2015.

[13] S. Garcia, D. Chatain, and J. P. Perin, Continuous Production of a Thin Ribbon of Solid Hydrogen, Laser Part. Beams 32, 569 (2014). 
[14] K. Jungwirth et al., The Prague Asterix Laser System, Phys. Plasmas 8, 2495 (2001).

[15] M. De Marco et al., Measurement of Electromagnetic Pulses Generated during Interactions of High Power Lasers with Solid Targets, J. Instrum. 11, C06004 (2016).

[16] E. Woryna, P. Parys, J. Wołowski, and W. Mróz, Corpuscolar Diagnostics and Processing Methods Applied in Investigations of Laser-Produced Plasma as a Source of Highly Ionized Ions, Laser Part. Beams 14, 293 (1996).

[17] D. Margarone et al. Full Characterization of LaserAccelerated Ion Beams Using Faraday Cup, Silicon Carbide, and Single-Crystal Diamond Detectors, J. Appl. Phys. 109, 103302 (2011); G. Bertuccio , D. Puglisi, L. Torrisi, and C. Lanzieri, Appl. Surf. Sci. 272, 128 (2013).

[18] J. Prokupek et al., Development and First Experimental Tests of Faraday Cup Array, Rev. Sci. Instrum. 85, 013302 (2014).

[19] T. Pisarczyk et al. Pre-Plasma Effect on Energy Transfer from Laser Beam to Shock Wave Generated in Solid Target, Phys. Plasmas 21, 012708 (2014); Space-Time Resolved Measurements of Spontaneous Magnetic Fields in LaserProduced Plasma, Phys. Plasmas 22, 102706 (2015).

[20] D. Klir, J. Kravarik, P. Kubes, K. Rezac, E. Litseva, K. Tomaszewski, L. Karpinski, M. Paduch, and M. Scholz, Fusion Neutron Detector for Time-of-Flight Measurements in Z-Pinch and Plasma Focus Experiments, Rev. Sci. Instrum. 82, 033505 (2011).

[21] D. Klir et al., Efficient Neutron Production from SubNanosecond Laser Pulse Accelerating Deuterons on Target Front Side, Phys. Plasmas 22, 093117 (2015).

[22] A. Kasperczuk and T. Pisarczyk, Optica Applicata XXXI, 571 (2001).

[23] J. Krasa, P. Parys, L. Velardi, A. Velyhan, L. Ryc, D. Della Side, and V. Nassisi, Time-of-Flight Spectra for Mapping of Charge Density of Ions Produced by Lasers, Laser Part. Beams 32, 15 (2014).

[24] S. J. Gitometer, R. D. Jones, F. Begay, A. W. Ehler, J. F. Kephart, and R. Kristal, Fast Ions and Hot Electrons in the Laser-Plasma Interaction, Phys. Fluids 29, 2679 (1986).

[25] D. Margarone et al., New Methods for High Current Fast Ion Beam Production by Laser-Driven Acceleration, Rev. Sci. Instrum. 83, $02 \mathrm{~B} 307$ (2012); Advanced Scheme for
High-Yield Laser Driven Nuclear Reactions, Plasma Phys. Controlled Fusion 57, 014030 (2015).

[26] R. Liska et al., in ALE Method for Simulations of Laser-Produced Plasmas, Finite Volumes for Complex Applications VI: Problems and Perspectives, edited by J. Fořt et al. (Springer, Berlin, 2011), Vol. 4, pp. 857-873.

[27] E. J. Caramana, D. E. Burton, M. J. Shashkov, and P. P. Whalen, The Construction of Compatible Hydrodynamics Algorithms Utilizing Conservation of Total Energy, J. Comput. Phys. 146, 227 (1998).

[28] R. Loubere and M. Shashkov, A Subcell Remapping Method on Staggered Polygonal Grids for ArbitraryLagrangian-Eulerian Methods, J. Comput. Phys. 209, 105 (2005).

[29] M. Hanfland, R. J. Hemley, and H. K. Mao, Novel Infrared Vibron Absorption in Solid Hydrogen at Megabar Pressures, Phys. Rev. Lett. 70, 3760 (1993).

[30] D. Haberberger, S. Tochitsky, F. Fiuza, C. Gong, R. A. Fonseca, L. O. Silva, W. B. Mori, and C. Joshi, Collisionless Shocks in Laser-Produced Plasma Generate Monoenergetic High-Energy Proton Beams, Nat. Phys. 8, 95 (2012).

[31] C. M. Brenner et al., High Energy Conversion Efficiency in Laser-Proton Acceleration by Controlling Laser-Energy Deposition onto Thin Foil Targets, Appl. Phys. Lett. 104, 081123 (2014).

[32] M. Roth et al. Fast Ignition by Intense Laser-Accelerated Proton Beams, Phys. Rev. Lett. 86, 436 (2001).

[33] S. Atzeni, M. Temporal, and J. J. Honrubia, A First Analysis of Fast Ignition of Precompressed ICF Fuel by LaserAccelerated Protons, Nucl. Fusion 42, L1 (2002); M. Temporal, J. J. Honrubia, and S. Atzeni, Numerical Study of Fast Ignition of Ablatively Imploded Deuterium-Tritium Fusion Capsules by Ultra-Intense Proton Beams, Phys. Plasmas 9, 3098 (2002).

[34] T. D. Arber et al., Contemporary Particle-in-Cell Approach to Laser-Plasma Modeling, Plasma Phys. Controlled Fusion 57, 113001 (2015).

[35] B. Rus et al., in Diode-Pumped High Energy and High Power Lasers; ELI: Ultrarelativistic Laser-Matter Interactions and Petawatt Photonics; and HiPER: the European Pathway to Laser Energy, SPIE Proceedings Vol. 8080 (SPIE-International Society for Optical Engineering, Bellingham, WA, 2011), p. 808010. 